\title{
Review of implementation strategies to change healthcare provider behaviour in the emergency department
}

Kerstin de Wit, MBChB, BSc, MD, MSc**‡; Janet Curran, PhD, RN${ }^{\S}$; Brent Thoma, MD, MA, MSc"; Shawn Dowling, MD**; Eddy Lang, BSc, MD**; Nebojsa Kuljic, BSc, MD candidate ${ }^{\dagger \dagger}$; Jeffrey J. Perry, $\mathrm{MD}, \mathrm{MSc}^{\ddagger \neq}$; Laurie Morrison, MD, $\mathrm{MSc}^{\S \S}$ on behalf of the CAEP Knowledge Translation Symposium Working Group

\section{ABSTRACT}

Objectives: Advances in emergency medicine research can be slow to make their way into clinical care, and implementing a new evidence-based intervention can be challenging in the emergency department. The Canadian Association of Emergency Physicians (CAEP) Knowledge Translation Symposium working group set out to produce recommendations for best practice in the implementation of a new science in Canadian emergency departments.

Methods: A systematic review of implementation strategies to change health care provider behaviour in the emergency department was conducted simultaneously with a national survey of emergency physician experience. We summarized our findings into a list of draft recommendations that were presented at the national CAEP Conference 2017 and further refined based on feedback through social media strategies.

Results: We produced 10 recommendations for implementing new evidence-based interventions in the emergency department, which cover identifying a practice gap, evaluating the evidence, planning the intervention strategy, monitoring, providing feedback during implementation, and desired qualities of future implementation research.

Conclusions: We present recommendations to guide future emergency department implementation initiatives. There is a need for robust and well-designed implementation research to guide future emergency department implementation initiatives.

\section{RÉSUMÉ}

Objectifs: II peut s'écouler passablement de temps avant que les progrès découlant de la recherche en médecine d'urgence trouvent application en pratique clinique, et la mise en œuvre d'interventions fondées sur des données probantes au service des urgences (SU) peut se révéler une tâche ardue. Le groupe de travail du symposium sur l'application des connaissances de I'Association canadienne des médecins d'urgence (ACMU) a élaboré un ensemble de recommandations concernant les pratiques exemplaires dans la mise en œuvre d'une nouvelle science aux services des urgences au Canada.

Méthode: L'équipe a procédé à une revue systématique des stratégies de mise en œuvre visant à changer le comportement des fournisseurs de soins de santé au SU, menée en parallèle avec une enquête nationale sur l'expérience des médecins d'urgence. Une liste de recommandations préliminaires reposant sur les résultats de la recherche a ensuite été dressée, puis présentée au congrès national de I'ACMU de 2017, après quoi il y a eu amélioration des recommandations d'après les observations recueillies à l'aide de stratégies fondées sur les médias sociaux.

Résultats: Le groupe de travail a élaboré un ensemble de 10 recommandations sur la mise en œuvre de nouvelles interventions fondées sur des données probantes au SU; elles portent sur la reconnaissance des lacunes en matière de pratiques, l'évaluation des données probantes; la planification des stratégies d'intervention, la surveillance et la collecte des réactions durant la mise en œuvre ainsi que sur les qualités que devraient présenter les recherches futures sur la mise en œuvre.

Conclusion: Le groupe a élaboré un ensemble de recommandations visant à guider les initiatives futures de mise en œuvre au SU. Pour ce faire, il faudra mener des recherches de mise en œuvre solides et bien conçues.

Keywords: knowledge translation, implementation science, emergency department

From the *Department of Medicine, McMaster University, Hamilton, ON; †Department of Emergency Medicine and; $¥ D e p a r t m e n t ~ o f ~ H e m a t o l o g y$, Hamilton Health Sciences, Hamilton, ON; §School of Nursing, Dalhousie University, Halifax, NS; ףDepartment of Emergency Medicine, University of Saskatchewan, Saskatoon, SK; **Department of Emergency Medicine, Cumming School of Medicine, University of Calgary, Alberta Health Services, Edmonton, AB; ††Queen's University, Kingston, ON; ¥¥Department of Emergency Medicine, University of Ottawa, Clinical Epidemiology Program, Ottawa Hospital Research Institute, Ottawa, ON; and §§Rescu, Li Ka Shing Knowledge Institute, St. Michael's Hospital, University of Toronto, Toronto, ON.

Correspondence to: Dr. Kerstin de Wit, Emergency Department, Hamilton General Hospital, 237 Barton St. East, Hamilton, ON L8L 2X2; Email: dewitk@mcmaster.ca. 


\section{INTRODUCTION}

\section{Knowledge translation}

Emergency medicine is a rapidly evolving field with consistently emerging practice-changing evidence. A critical issue in our field is that advances in research are often slow in making their way into practice. ${ }^{1}$ Impacts demonstrated by science often do not result in changes in bedside care, or in improving patient outcomes. Implementation science is the study of methods to promote the uptake of research findings in routine health care in clinical, organizational, or policy contexts. ${ }^{2}$ In Canada, this is often known as knowledge translation, or KT.

\section{Choosing Wisely Canada}

Emergency physicians are being asked to be more efficient and effective in the care that they provide. Campaigns such as Choosing Wisely $\mathrm{Canada}^{3}$ are dedicated to highlighting the numerous areas where there are opportunities to eliminate low-value care. Some would argue that the greatest challenge facing healthcare is not generating new knowledge through research but identifying optimal methods of physician and system-level uptake when evidence is ready for implementation.

\section{Implementing new practice}

Barriers to implementation and achieving enduring change in clinical practice exist at the patient, provider, departmental, and institutional levels. Implementation is contingent on three key sources of change, which come with their own unique challenges: knowledge, attitudes, and behaviour. ${ }^{4}$ Knowledge acquisition can be challenging because of the volume of information, the time needed to stay informed, and the barriers to accessing educational resources. Because implementation is ultimately a local activity, an emergency department (ED) must identify its own knowledge and behaviour gaps. Robust data collection and monitoring to demonstrate a practice gap are central to changing attitudes, but these are also labour intensive. Finally, changing behaviour is difficult to achieve, with inertia and external pressures promoting the status quo.

While implementation science has gained traction as a focus of research, implementation occurs within a local and political context that should take into account local barriers and enablers for change. ${ }^{4}$ Department or systemwide change requires champions and committed hospital resources. New protocols ideally incorporate patient preferences or expectations as well as cost-effectiveness, medicolegal, or regulatory considerations. ${ }^{5}$

\section{Aim}

Evidence-based emergency medicine care requires evidence-based implementation strategies. Recognizing the challenging environment unique to emergency medicine, the working group's aim was to develop recommendations for the robust and reliable implementation of evidence-based practices in Canadian EDs.

\section{METHODS}

The Canadian Association of Emergency Physicians (CAEP) working group consisted of five practicing emergency medicine physicians who are active implementation scientists, a clinical nurse implementation scientist, and a medical student.

There were three parts to this project. Firstly, we conducted a systematic review of implementation strategies to change healthcare provider behaviour in the ED setting. Secondly, we conducted a national survey of emergency physicians to gain insight into different strategies used to plan, implement, and evaluate new interventions within the ED. Thirdly, we summarized our findings into a list of draft recommendations that were presented at the national CAEP Conference 2017 and online for public comments through social media.

\section{Systematic review}

A systematic review of literature was conducted (by author JC) to identify, appraise, and synthesize the best available evidence on implementation strategies that have been evaluated in ED settings to change healthcare provider behaviour. The review will be published in full separately. We included studies that evaluated implementation strategies as described in the Cochrane Effective Practice and Organization of Care (EPOC) Taxonomy. ${ }^{6}$ The outcomes of interest included change in provider behaviour or change in patient behaviour. The search strategy was developed in collaboration with a library scientist for the following databases: MEDLINE, CINAHL, Embase, and Cochrane CENTRAL for primary studies complemented by a manual search of the 
past 5 years of journals, Annals of Emergency Medicine, CFEM, and Implementation Science.

\section{Online survey}

An online questionnaire (online Supplement 1) was sent to all CAEP members on January 24, 2017, with two further reminders. An additional, personalized invite reminder was sent from the authors to each nonresponding CAEP member who had registered himself or herself with CAEP as an emergency medicine leader, educator, or researcher. Only respondents who answered that they had ED implementation experience or had been a research study primary investigator were asked to complete the survey.

The questionnaire branched into focused lines of questioning depending on how the responders identified themselves. Educators and clinicians were asked about methods for communicating new strategies, changing physician behaviour, identifying the barriers to change, and measuring the impact of the change. Leaders answered questions about the implementation techniques that they used, which techniques they recommended, the importance of timely implementation, and the effectiveness of implementation strategies. Researchers were questioned about the ways that they communicated their study findings and how they changed local physician behavior based on the results of their research findings.

Categorical responses were summarized using proportions, and free text responses underwent thematic analysis by two group members (NK and $\mathrm{KdW}$ ).

\section{Development of CAEP KT Recommendations}

The KT working group met weekly between April and June 2017 to discuss the key findings of the survey and systematic review, and to draft an initial 10 recommendations through an iterative process. The systematic review, survey results, and draft list of recommendations were presented to an audience of peers at the 2017 conference of the CAEP Academic Symposium in Whistler, British Columbia. They were simultaneously posted online (www.canadiem.org/ knowledge-translation-recommendations), and comments from the public were encouraged through the Fluid Surveys ${ }^{\mathrm{TM}}$ interface. The working group used this feedback to derive the final list of recommendations.

\section{RESULTS}

\section{Systematic review results}

The results of the systematic review can be found in the online Supplements 2 and 3 and included 33 studies. ${ }^{7-39}$ Most included studies were randomized controlled trials $(n=32)$ conducted in urban academic emergency practice settings $(n=27)$ in the United States of America $(\mathrm{n}=21)$ and published between 2000 and 2017 $(\mathrm{n}=33)$.

A total of nine different implementation strategies were used; reminders (manual or computerized interventions that prompt healthcare workers to perform an action) $(\mathrm{n}=22)$, educational meetings (courses, workshops, or other educational meetings) $(\mathrm{n}=15)$, educational materials (distribution of educational materials to individuals or groups to support clinical care) $(\mathrm{n}=11)$, and clinical practice guidelines (systematically developed statements to assist healthcare providers and patients to decide on appropriate healthcare for specific clinical circumstances) $(n=7)$ were the most common implementation strategies used. Most studies (20/33) included more than one implementation strategy, and some type of educational strategy was included in the majority of these multifaceted interventions $(17 / 20)$.

Primary outcomes in the included studies incorporated a range of healthcare provider level outcomes (e.g., proportion of $\mathrm{X}$-rays ordered, knowledge, use of clinical practice guideline), patient level outcomes (e.g., proportion of patients referred to the stroke unit, patient satisfaction), and health system level outcomes (e.g., level of stay, time to disposition decision).

\section{Survey results}

In all, 455 emergency physicians replied to the survey out of a total CAEP membership of 2,500 (response rate of $18 \%$ ). Only 301 responders had experience in implementation or were study primary investigators; these included 119 clinicians, 76 leaders, 59 educators, and 47 researchers (see online Supplement 4 for details of responders).

Eighty percent (61/76) of ED leaders felt that timely practice change after the publication of new guidelines was very important, and 70\% (53/76) thought that implementation efforts in their department were "somewhat effective." Most respondents (148/240) 
felt that it was difficult to measure the impact of an implementation strategy.

Eighty-five percent (33/39) of researchers thought that journals had a role to play in the dissemination of research study results, over and above the publication of a manuscript. Thirty-one percent of researchers (12/39) reported a journal where they had published had aided in knowledge dissemination of the findings, for example, through social media, the press, or broadcasting of interviews.

The most common methods of communicating new guidelines or pathways were ED rounds (departmental educational meetings) (262/301) and emails (228/301). Other routes included presenting at multidisciplinary rounds (116/301) and meetings with hospital administration (85/301). A few reported publishing in local news media (34/301), using Facebook or Twitter (49/301), links to blogs (32/301), promoting a new app $(41 / 301)$, or using infographics $(65 / 301)$.

Free text analysis resulted in the identification of 13 themes, outlined in Table 1 . The coding scheme that relates each code to a theme can be found in the online Supplement 5.

\section{Feedback from the CAEP academic symposium}

The draft recommendations, feedback, and edited final recommendations are outlined in Box 1 and Supplementary Material 6.

\section{SUMMARY OF RECOMMENDATIONS}

Recommendation 1: Before implementing change, ensure that your physician group understands the evidence, acknowledges a gap in local practice, and is willing to change. Agree on group-level targets before an implementation program starts.

At a basic level, the group of clinicians involved in implementing a change of practice must be convinced by the evidence supporting it. This will involve an examination of local data and available published guidelines and/or best practice evidence. Physician buy-in at the start increases uptake of the new intervention. Group agreement on the aim of the implementation and the outcomes that they will work towards will help facilitate change. For example, a group may decide that an intervention to improve the diagnosis of pulmonary embolism should result in fewer missed cases of pulmonary embolism.
Recommendation 2: Consider following guidelines rather than implementing an intervention based on a body of work that has not been adequately validated. Both premature and delayed implementation can have negative consequences.

Novel research with dramatic results or in particularly interesting areas of practice can often be implemented prematurely. This can be problematic because findings from these studies may eventually turn out to be an overestimation of the reported effect or even incorrect. We recommend following expert guidelines on the topic rather than implementing an intervention that has not yet been validated in more than one trial. Further, a delay in implementing evidence-based guidelines may deprive patients of beneficial diagnostic or therapeutic options. Therefore, a balance is needed so that new evidence is implemented soon after validation has occurred or the evidence has been adopted by expert guidelines.

\section{Recommendation 3: Practice changing interventions should target clinical problems where care can be significantly improved.}

Interventions require effort and investment and should be targeted to have the most impact. It is necessary to review current local practice (for example, by chart review) to identify where practice diversifies from the best evidence. Any intervention should be aimed at reducing these specific gaps between the evidence and clinical practice.

\section{Recommendation 4: Develop implementation strategies that address local circumstances.}

Local culture and circumstance can play a significant role in the success or failure of an implementation initiative. Although it may be tempting to replicate implementation strategies that have been shown to be successful at other sites, consideration must be given to the environment in which the implementation is occurring with modifications to address the local barriers to change.

\section{Recommendation 5: Effective practice changing interventions in the ED setting may include, but are not} limited to, institutional support, local champions, standardized order sets, education, audit, and feedback.

Implementation strategies composed of single interventions can be easily ignored and are rarely successful. To maximize the likelihood of adoption, consideration 
Recommendations for implementation strategies in the ED

\begin{tabular}{|c|c|}
\hline Theme & Description \\
\hline $\begin{array}{l}\text { Choosing what to } \\
\text { implement }\end{array}$ & Choosing to implement the right knowledge when there is sufficient evidence of benefit and harms. \\
\hline Assessing barriers & $\begin{array}{l}\text { Anticipate reasons for potential disengagement of the physician group. Identify barriers to outcome } \\
\text { measurement. Ensuring adequate resources: funds, expertise, time. }\end{array}$ \\
\hline Group buy-in & $\begin{array}{l}\text { Ensure that the physicians value the change. Consider credibility of the evidence, review data on current local } \\
\text { clinical outcomes, and work towards shared group values. }\end{array}$ \\
\hline Leadership & Consider several types of leaders: management, local champions, and experts in implementation science. \\
\hline Multidisciplinary team & $\begin{array}{l}\text { Make your implementation team as large as possible to include other disciplines, other healthcare workers, } \\
\text { policymakers, educators, and researchers. }\end{array}$ \\
\hline Engagement & $\begin{array}{l}\text { Engage your group in many ways: involve them from the outset, give them opportunities to question, share, and } \\
\text { co-create strategies. }\end{array}$ \\
\hline Targeted dialogue & $\begin{array}{l}\text { Discuss the intervention/evidence in meetings, journal club, rounds, focus groups, morbidity and mortality } \\
\text { rounds, as well as everyday conversation. }\end{array}$ \\
\hline $\begin{array}{l}\text { Choose implementation } \\
\text { strategies }\end{array}$ & $\begin{array}{l}\text { There are many strategies to encourage change: change the behaviour of opinion leaders first, lead by example, } \\
\text { educate nurses and patients, make physicians accountable by benchmarking performance, incentivize the new } \\
\text { behaviours, automate the new strategy to make it the easiest option, link the change to professionalism, and } \\
\text { many more. }\end{array}$ \\
\hline $\begin{array}{l}\text { Simplifying } \\
\text { implementation }\end{array}$ & $\begin{array}{l}\text { Develop care pathways / order sets / care bundles. When appropriate, make them simple, automated, easy to } \\
\text { access. Embed in electronic health systems where possible. }\end{array}$ \\
\hline IT systems & $\begin{array}{l}\text { Ensure that electronic pathways are user friendly and easy to use. Work with IT support to produce integrated } \\
\text { pathways that can also be used to assess electronic data for compliance and outcomes. }\end{array}$ \\
\hline Education & $\begin{array}{l}\text { Education should be multifaceted. Embed within the resident curriculum, teach at the bedside, offer in-person or } \\
\text { E-learning courses, give clinical demonstrations, use in simulation, create or share infographics, videos, } \\
\text { podcasts, blogs. }\end{array}$ \\
\hline Repetition & $\begin{array}{l}\text { Repeat the message at every opportunity. Talk about the change in rounds, journal club, new letters, emails, } \\
\text { social media, and in the corridors. }\end{array}$ \\
\hline $\begin{array}{l}\text { Active monitoring and } \\
\text { feedback }\end{array}$ & $\begin{array}{l}\text { Integrate the change into a quality improvement project. Measure the outcomes (both clinical outcomes and } \\
\text { physician compliance). Plan how you will do this before starting. Feed the outcomes back to the physician } \\
\text { group. }\end{array}$ \\
\hline
\end{tabular}

should be given to strategies for communicating, facilitating, and tracking the desired change at multiple levels. ED workflow should not be disrupted by the new procedures and should be provided with adequate support to facilitate the change. Constructive feedback on both compliance and clinical outcomes is critical to ensure continued buy-in.

\section{Recommendation 6: Involve all relevant stakeholders, including physicians, nurses, patients, administrators, and allied healthcare providers. A team-based approach to developing, integrating, and supporting an implementation strategy is the key to success.}

Implementation strategies have the greatest potential for adoption and sustainability if all of the relevant stakeholders are involved from the beginning of the project. The degree of involvement and the number of participants will depend on availability and resources but, at the bare minimum, should include the stakeholders whom are most directly impacted by the project. This engagement achieves multiple objectives, including buy-in from the stakeholders, the identification of local barriers and facilitators, the incorporation of differing values/perspectives, the development of novel local solutions, and the identification of local champions.

Recommendation 7: When possible, measure the impact of the implementation strategy by tracking compliance, following directly and indirectly related patient-oriented outcomes, and investigating unanticipated consequences.

To determine the impact of the implementation strategy, it is critical to measure both process and outcomes 
Box 1. CAEP Knowledge Translation Recommendations

- Before implementing change, ensure your physician group understands the evidence, acknowledges a gap in local practice and is willing to change. Agree on group-level targets before an implementation program starts.

- Consider following guidelines rather than implementing an intervention based on a body of work that has not been adequately validated. Both premature and delayed implementation can have negative consequences.

- Practice changing interventions should target clinical problems where care can be significantly improved.

- Develop implementation strategies that address local circumstances.

- Effective practice change interventions may include, but are not limited to, institutional support, local champions, standardized order sets, education, audit and feedback.

- Involve all relevant stakeholders - including physicians, nurses, patients, administrators, and allied health care providers. A team based approach to developing, integrating, and supporting an implementation strategy is the key to success.

-When possible, measure the impact of the implementation strategy by tracking compliance, following directly and indirectly related patient-oriented outcomes, and investigating unanticipated consequences.

- Make successful interventions sustainable by ensuring that the relevant business plan provides adequate resources and that key tasks are incorporated into relevant job descriptions, roles, and responsibilities.

- Formal ED implementation studies/trials should be rigorously designed and include assessments of individual provider, patient and system level barriers prior to designing and implementing the intervention.

- Protocols of ED implementation studies should be registered in an appropriate

measures. Process measures, such as compliance with the intervention, are important to ensure that improved outcomes are a function of a change in process and identifies when the implementation strategy is achieving adequate penetration. Compliance is best measured by mapping out the process of care step by step and a priori defining a simple measure of compliance at each step. Measurement of patient-oriented outcomes (rather than surrogate outcomes) ensures the intervention results in improved clinical outcomes. Defining patient-oriented outcomes is best achieved by rank ordering the outcomes based on importance and ease of capture or feasibility. In other words, at the top of the list would be items that are critically important and simple to capture. Measuring negative or unanticipated consequences is important to ensure that deleterious consequences are not occurring.
Recommendation 8: Make successful interventions sustainable by ensuring that the relevant business plan provides adequate resources and that key tasks are incorporated into relevant job descriptions, roles, and responsibilities.

One of the challenges with successful interventions can be sustaining the implemented change. Without a plan, the new intervention will slowly stop being used. To enable continual and persistent change, the project should have a business plan where longitudinal roles are clearly defined. To ensure sustainability, different aspects of the intervention are incorporated into appropriate personnel roles (frequently clinicians, registered nurses, administrators, and business clerks). This makes continued behaviour change an attractive option because the workload is dispersed and the process is automated.

Recommendation 9: Formal ED implementation studies/ trials should be rigorously designed and include assessments of individual provider, patient, and system level barriers prior to designing and implementing the intervention.

There is a clear need for high quality, rigorously designed implementation science trials that specifically address the unique challenges in the ED. These studies should use an evidence-based approach to developing their implementation strategy, for example, by evaluating pre-existing barriers in multiple EDs, before developing an implementation strategy that targets these barriers.

Recommendation 10: Protocols of ED implementation studies should be registered in an appropriate database. Registration information should outline the rationale for the intervention components and assumptions about how the implementation strategy will work.

Registering of implementation science clinical trials (i.e., clinical trials.gov) is an important step to minimize the risk of publication bias, selective reporting of results and to ensure the transparency of the research protocols.

\section{CONCLUSION}

We have made recommendations for future ED implementation strategies, based on a systematic review of existing literature and the experience of Canadian emergency physicians who have lead ED practice change. The recommendations cover the identification 
of the evidence-practice gap, planning of the implementation strategy, measurement of outcomes, and planning sustainability of the intervention. Future high quality $\mathrm{ED}$ implementation research is needed to clearly identify optimal implementation strategies.

Acknowledgements: We thank Kelly Wyatt, Jennifer Artz, and Shanna Scarrow (CAEP) for their help in administering the survey and scheduling our group meetings.

Competing interests: None declared.

\section{SUPPLEMENTARY MATERIAL}

To view supplementary material for this article, please visit https://doi.org/10.1017/cem.2017.432

\section{REFERENCES}

1. Gaddis G, Greenwald P, Huckson S. Toward improved implementation of evidence-based clinical algorithms: clinical practice guidelines, clinical decision rules, and clinical pathways. Acad Emerg Med 2007;14:1015-22.

2. CAEP Academic Symposium. Better research through engagement, implementation, and knowledge translation; 2017. Available at: http://caep.ca/AcademicSymposia $\% 20$ (accessed August 2017).

3. Cheng AHY, Campbell S, Chartier LB, et al. Choosing Wisely Canada: five tests, procedures, and treatments to question in emergency medicine. CFEM 2017;19:S9-17.

4. Tavender EJ, Bosch M, Fiander M, et al. Implementation research in emergency medicine: a systematic scoping review. EM7 2016;33:652-9.

5. Lang ES, Wyer PC, Haynes RB. Knowledge translation: closing the evidence-to-practice gap. Ann Emerg Med 2007;49:355-63.

6. Effective Practice and Organisation of Care (EPOC). EPOC taxonomy; 2015. Available at: http://epoc.cochrane.org/sites/ epoc.cochrane.org/files/public/uploads/epoc_taxonomy_13. 12.16.pdf (accessed August 2017).

7. Auleley GR, Ravaud P, Giraudeau B, et al. Implementation of the Ottawa ankle rules in France. A multicenter randomized controlled trial. FAMA 1997;277(24):1935-9.

8. Baumann BM, McCans K, Stahmer SA, et al. Volumetric bladder ultrasound performed by trained nurses increases catheterization success in pediatric patients. Am 7 Emerg Med 2008;26(1):8-23.

9. Becerra-Camargo J, Martinez-Martinez F, Garcia-Jimenez E. The effect on potential adverse drug events of a pharmacist-acquired medication history in an emergency department: a multicentre, double-blind, randomised, controlled, parallel-group study. BMC Health Serv Res 2015;15:337.

10. Bullard MJ, Meurer DP, Colman I, et al. Supporting clinical practice at the bedside using wireless technology. Acad Emerg Med 2004;11(11):1186-92.
11. Campbell JC, Coben JH, McLoughlin E, et al. An evaluation of a system-change training model to improve emergency department response to battered women. Acad Emerg Med 2001;8(2):131-8.

12. De Luca A, Toni D, Lauria L, et al. An emergency clinical pathway for stroke patients - results of a cluster randomised trial (isrctn41456865). BMC Health Serv Res 2009;9:14.

13. Derksen RJ, Bakker FC, de Lange-de Klerk ESM, et al. Specialized emergency nurses treating ankle and foot injuries: a randomized controlled trial. Am 7 Emerg Med 2007;25(2):144-51.

14. De Vos-Kerkhof E, Nijman RG, Vergouwe Y, et al. Impact of a clinical decision model for febrile children at risk for serious bacterial infections at the emergency department: a randomized controlled trial. PLoS One 2015;10(5):e0127620.

15. Dexheimer JW, Abramo TJ, Arnold DH, et al. Implementation and evaluation of an integrated computerized asthma management system in a pediatric emergency department: a randomized clinical trial. Int 7 Med Inform 2014;83(11):805-13.

16. Dexheimer JW, Abramo TJ, Arnold DH, et al. An asthma management system in a pediatric emergency department. Int 7 Med Inform 2013;82(4):230-8.

17. Guenther E, Olsen C, Keenan H, et al. Randomized prosective study to evaluate child abuse documentation in the emergency department. Acad Emerg Med 2009; 16(3):249-57.

18. Holmes JF, Freilich J, Taylor SL, et al. Electronic alerts for triage protocol compliance among emergency department triage nurses: a randomized controlled trial. Nurse Res 2015;64(3):226-30.

19. Kinsman LD, Rotter T, Willis J, et al. Do clinical pathways enhance access to evidence-based acute myocardial infarction treatment in rural emergency departments? Aust $\mathcal{7}$ Rural Health 2012;20(2):59-66.

20. Kozer E, Scolnik D, MacPherson A, et al. Using a preprinted order sheet to reduce prescription errors in a pediatric emergency department: a randomized, controlled trial. Pediatrics 2005;116(6):1299-302.

21. Linn BS. Continuing medical education. Impact on emergency room burn care. FAMA 1980;244(6):565-70.

22. Marill KA, Gauharou ES, Nelson BK, et al. Prospective, randomized trial of template-assisted versus undirected written recording of physician records in the emergency department. Ann Emerg Med 1999;33(5):500-9.

23. Metlay JP, Camargo CAJ, MacKenzie T, et al. Clusterrandomized trial to improve antibiotic use for adults with acute respiratory infections treated in emergency departments. Ann Emerg Med 2007;50(3):221-30.

24. Meurer WJ, Frederiksen SM, Xu Z, et al. Improvement in emergency physician stroke thrombolysis knowledge: the increasing tPA stroke treatment through interventional behavior change tactics (INSTINCT) trial. Stroke 2011;42(3):e147.

25. Raja AS, Ip IK, Dunne RM, et al. Effects of performance feedback reports on adherence to evidence-based guidelines in use of CT for evaluation of pulmonary embolism in the emergency department: a randomized trial. A7R Am 7 Roentgenol 2015;205(5):936-40.

26. Rathlev N, Almomen R, Deutsch A, et al. Randomized controlled trial of electronic care plan alerts and resource 
utilization by high frequency emergency department users with opioid use disorder. West 7 Emerg Med 2016;17(1): 28-34.

27. Smeekens AEFN, Broekhuijsen-van Henten DM, Sittig JS, et al. Successful e-learning programme on the detection of child abuse in emergency departments: a randomised controlled trial. Arch Dis Child 2011;96(4):330-4.

28. Stiell IG, Clement CM, Grimshaw J, et al. Implementation of the Canadian C-spine rule: Prospective 12 centre cluster randomised trials. BM7 29; 339:b4146.

29. Stiell IG, Clement CM, Grimshaw JM, et al. A prospective cluster-randomized trial to implement the Canadian CT Head Rule in emergency departments. CMAJ 2010;182 (14):1527-32.

30. Stiell IG, Wells GA, Hoag RH, et al. Implementation of the Ottawa Knee Rule for the use of radiography in acute knee injuries. 7AMA 1997;278(23):2075-9.

31. Taylor DM, Fatovich DM, Finucci DP, et al. Best-practice pain management in the emergency department: a clusterrandomised, controlled, intervention trial. Emerg Med Australas 2015;27(6):549-57.

32. Terrell KM, Perkins AJ, Dexter PR, et al. Computerized decision support to reduce potentially inappropriate prescribing to older emergency department patients: a randomized, controlled trial. 7 Am Ger Soc 2009;57(8): 1388-94.

33. Terrell KM, Perkins AJ, Hui SL. Computerized decision support for medication dosing in renal insufficiency: a randomized, controlled trial. Ann Emerg Med 2010;56(6): 623-9.

34. Walensky RP, Reichmann WM, Arbelaez C, et al. Counselor- versus provider-based HIV screening in the emergency department: results from the universal screening for HIV infection in the emergency room (USHER) randomized controlled trial. Ann Emerg Med 2011;58 (1 Suppl 1):S126-32.

35. Yealy DM, Auble TE, Stone RA, et al. Effect of increasing the intensity of implementing pneumonia guidelines: a randomized, controlled trial. Ann Intern Med 2005;143 (12):881-94.

36. Dunn C, Zatzick D, Donovan D, et al. Can front-line trauma center providers be trained in and sustain alcohol motivational interviewing skills? Results from a multi-site randomized trial. Alcobol Clin Exp Res 2013;37:262A.

37. Fitzgerald M, Cameron P, Mackenzie C, et al. Trauma resuscitation errors and computer-assisted decision support. Arch Surg 2011;146(2):218-25.

38. Geurts D, de Vos-Kerkhof E, Polinder S. Implementation of clinical decision support in young children with acute gastroenteritis: a randomized controlled trial at the emergency department. Eur $\mathcal{f}$ Pediatr 2017;176(2): 173-81.

39. Goyal MK, Fein JA, Badolato GM, et al. A computerized sexual health survey improves testing for sexually transmitted infection in a pediatric emergency department. 7 Pediatr 2016;183:147-52. 